\title{
A converse comparison theorem for backward stochastic differential equations with jumps*
}

\author{
Xavier De Scheemaekere ${ }^{\dagger}$
}

May 2011

\begin{abstract}
This paper establishes a converse comparison theorem for real-valued decoupled forward backward stochastic differential equations with jumps.

$M S C$ 2010: primary 60H10

Keywords: Backward stochastic differential equation with jumps; Comparison theorem; Converse comparison theorem.
\end{abstract}

\section{Introduction}

A backward stochastic differential equation (BSDE) is an equation of the type

$$
Y_{t}=\xi_{T}+\int_{t}^{T} g\left(s, Y_{s}, Z_{s}\right) d s-\int_{t}^{T} Z_{s} d W_{s}
$$

where $\xi$ is called the terminal condition, $T$ the terminal time, $g$ the generator, and the pair of processes $(Y, Z)$ the solution of the equation. In 1990, Pardoux and Peng (8) proved that there exists a unique adapted and square integrable solution to BSDE (11) as soon as the generator is Lipschitz with respect to $y$ and $z$ and the terminal condition is square integrable. Since then, BSDE theory has become an important field of research, with applications, e.g., in stochastic optimal control, mathematical finance or partial differential equations (PDEs).

An important feature of BSDE theory is the comparison theorem (Peng (9), El Karoui et al. (5)), which plays the same role as the maximum principle for PDEs. The comparison theorem allows to compare the solutions of two

*This paper is based on a homonymous paper published in Statistics and Probability Letters 81, 298-301, 2011, which contains an error in the proof of the main theorem. It presents a similar result, but for a more restricted class of equations, so that the error is now fixed. The author thank Monique Jeanblanc for pointing out the error and for fruitful discussions about how to fix it.

${ }^{\dagger}$ F.R.S.-F.N.R.S. Research Fellow; Centre Emile Bernheim, Solvay Brussels School of Economics and Management, Université libre de Bruxelles (ULB); Postal address: Av. F.D. Roosevelt, 50, CP 145/1, 1050 Brussels, Belgium ; Tel.: +32.2.650.39.59; E-mail address: xdeschee@ulb.ac.be. 
real-valued BSDEs whenever we can compare the terminal conditions and the generators. The converse, however, is not true in general. Hence, converse comparison theorems for BSDEs have been concerned with the following question: If one can compare the solutions of two real-valued BSDEs with the same terminal condition, for all terminal conditions, can one compare the generators?

Previous works on the subject (Chen (3), Briand et al. (2), Coquet et al. (4), Jiang (6),(7)) deal with classical BSDEs (i.e., without jumps). This paper provides a converse comparison theorem for class of BSDEs with jumps, namely infinite horizon decoupled forward backward stochastic differential equations with jumps.

\section{BSDEs with jumps}

\subsection{Notation and assumptions}

Suppose that $W_{t}^{\top}=\left(W_{t}^{1}, \ldots, W_{t}^{d}\right), t \geq 0$, is a $d$-dimensional standard Brownian Motion and $K^{\top}(t)=\left(K_{1}(t), \ldots, K_{l}(t)\right), t \geq 0$, is an $l$-dimensional stationary Poisson point process taking values in a measurable space $(B, \mathbf{B})$, where $B=\mathbb{R}_{0}$ is equipped with its Borel field $\mathbf{B}$. Denote by $\mu^{\top}(d t, d e)=\left(\mu_{1}(d t, d e), \ldots, \mu_{l}(d t, d e)\right)$ the Poisson random measure induced by $K(\cdot)$ with compensator $\lambda^{\top}(d e) d t=$ $\left(\lambda_{1}(d e), \ldots, \lambda_{l}(d e)\right) d t$ such that $\left\{\widetilde{\mu}_{i}([0, t], A)=\left(\mu_{i}-\lambda_{i}\right)([0, t], A)\right\}_{t \geq 0}$ is a martingale for all $A \in \mathbf{B}$ satisfying $\lambda_{i}(A)<\infty, i=1, \ldots, l$. $\lambda_{i}$ is assumed to be a $\sigma$-finite measure on $(B, \mathbf{B})$.

Let $\left(\Omega, \mathbf{F},\left(F_{t}\right)_{t \geq 0}, P\right)$ be a complete and standard measurable probability space equipped with a filtration denoted as

$$
\left(F_{t}\right)_{t \geq 0}=\left(\sigma\left[W_{s} ; s \leq t\right] \vee \sigma[\mu((0, s], A) ; s \leq t, A \in \mathbf{B}] \vee N\right)_{t \geq 0},
$$

where $N$ is all the $P$-null sets. It is a complete right-continuous filtration. Let $\tau$ be a (possibly infinite) $\left(F_{t}\right)_{t \geq 0}$-stopping time and define the following spaces:

- $S^{2}$ denotes the set of all $F_{t}$-progressively measurable RCLL processes $Y$ valued in $\mathbb{R}^{n}$ such that $E\left[\sup _{0 \leq t \leq \tau}\left|Y_{t}\right|^{2}\right]<\infty$;

- $L^{2}(W)$ denotes the set of all predictable 1 processes $Z$ valued in $\mathbb{R}^{n \times d}$ such that $E\left[\int_{0}^{\tau}\left|Z_{t}\right|^{2} d t\right]<\infty$;

- $L^{2}(\widetilde{\mu})$ denotes the set of all $\mathbf{P} \otimes \mathbf{B}$-measurable processes $U_{t}(e)$ valued in $\mathbb{R}^{n \times l}$ such that

$$
E\left[\int_{0}^{\tau} \int_{B}\left|U_{t}(e)\right|^{2} \lambda(d e) d t\right]:=E\left[\int_{0}^{\tau}\left\|U_{t}(\cdot)\right\|^{2} d t\right]<\infty
$$

where $\mathbf{P}$ denotes the $\sigma$-algebra generated by all predictable subsets;

\footnotetext{
${ }^{1}$ By predictable, we mean predictable with respect to the filtration $\left(F_{t}\right)_{0 \leq t \leq \tau}$.
} 
- $L^{2}\left(B, \mathbf{B}, \lambda ; \mathbb{R}^{n \times l}\right)$ denotes the set of all $\mathbf{B}$-measurable functions $\Phi(\cdot)$ valued in $\mathbb{R}^{n \times l}$ such that

$$
\|\Phi\|^{2}=\int_{B}|\Phi(e)|^{2} \lambda(d e)<\infty
$$

The (adapted) solution of a random horizon BSDE with jumps is defined as a triple of processes $\left(Y_{t}, Z_{t}, U_{t}\right), t \in[0, \tau]$, belonging to $S^{2} \times L^{2}(W) \times L^{2}(\widetilde{\mu})$ such that

$$
Y_{t}=\xi_{\tau}+\int_{t \wedge \tau}^{\tau} f\left(\omega, s, Y_{s}, Z_{s}, U_{s}\right) d s-\int_{t \wedge \tau}^{\tau} Z_{s} d W_{s}-\int_{t \wedge \tau}^{\tau} \int_{B} U_{s}(e) \widetilde{\mu}(d s, d e),
$$

where the terminal condition $\xi \in L^{2}\left(\Omega, F_{\tau}, P\right)$ and the generator $f: \Omega \times$ $[0, \infty) \times \mathbb{R}^{n} \times \mathbb{R}^{n \times d} \times L^{2}\left(B, \mathbf{B}, \lambda ; \mathbb{R}^{n \times l}\right) \rightarrow \mathbb{R}^{n}$ is $\mathbf{P} \otimes \mathbf{B}\left(\mathbb{R}^{n}\right) \otimes \mathbf{B}\left(\mathbb{R}^{n \times d}\right) \otimes$ $\mathbf{B}\left(L^{2}\left(B, \mathbf{B}, \lambda ; \mathbb{R}^{n \times l}\right)\right)$-measurable.

We will denote by $Y_{t}\left(f, \xi_{\tau}\right)$ the first component of the solution of BSDE (2). In the sequel, we consider the following assumptions on generator $f$ :

$$
E\left[\int_{0}^{\tau}|f(\omega, t, 0,0,0)|^{2} d t\right]<\infty
$$

(A2) $P-$ a.s., for any $Y, Y^{\prime} \in \mathbb{R}^{n}, Z, Z^{\prime} \in \mathbb{R}^{n \times d}, U, U^{\prime} \in L^{2}\left(B, \mathbf{B}, \lambda ; \mathbb{R}^{n \times l}\right)$, $t \geq 0$

$$
\begin{aligned}
\left|f(\omega, t, Y, Z, U)-f\left(\omega, t, Y^{\prime}, Z^{\prime}, U^{\prime}\right)\right| \leq & u_{1}(t)\left|Y-Y^{\prime}\right| \\
& +u_{2}(t)\left(\left|Z-Z^{\prime}\right|+\left\|U-U^{\prime}\right\|\right),
\end{aligned}
$$

where $u_{1}(t)$ and $u_{2}(t)$ are nonnegative, deterministic functions satisfying

$$
\int_{0}^{\infty} u_{1}(t) d t+\int_{0}^{\infty} u_{2}(t)^{2} d t<\infty
$$

(A3) $P-$ a.s, $\forall(Y, Z, U) \in \mathbb{R}^{n} \times \mathbb{R}^{n \times d} \times L^{2}\left(B, \mathbf{B}, \lambda ; \mathbb{R}^{n \times l}\right), t \rightarrow f(\omega, t, Y, Z, U)$ is continuous in $t \in[0, \tau]$.

(A4) $P-a . s, \forall Y \in \mathbb{R}^{n}, \forall Z \in \mathbb{R}^{n \times d}, \forall U, U^{\prime} \in L^{2}\left(B, \mathbf{B}, \lambda ; \mathbb{R}^{n \times l}\right)$, we have

$$
\left|f(\omega, t, Y, Z, U)-f\left(\omega, t, Y, Z, U^{\prime}\right)\right| \leq\left|\int_{B}\left(U(e)-U^{\prime}(e)\right) \bar{\gamma}_{t}(\omega, e)^{\top} \lambda(d e)\right|,
$$

where $\bar{\gamma}: \Omega \times[0, \infty) \times B \rightarrow \mathbb{R}^{n \times l}$ is $\mathbf{P} \otimes \mathbf{B}$-measurable and satisfies

$\int_{B}\left|\bar{\gamma}_{t}(\omega, e)\right|^{2} \lambda(d e) \leq u_{2}(t)^{2}$, and where $\bar{\gamma}_{t}^{i}(\omega, e), i=1, \ldots, l$, is the $i$ th component of $\bar{\gamma}_{t}(\omega, e)$ satisfying $\bar{\gamma}_{t}^{i}(\omega, e) \geq-1, P-a . s$. 


\subsection{Preliminary results}

This section presents existence, uniqueness and comparison results for adapted solutions to BSDEs with jumps and with random terminal times.

Theorem 1 (Yin and Situ (13)) Let $\xi_{\tau}$ and $f$ be the terminal condition and the generator of BSDE (2), respectively, and let $f$ satisfy (A1) and (A2). Then there exists a unique solution to BSDE (2).

In preparation of the main theorem, we recall the comparison theorem for one-dimensional BSDEs with jumps. Therefore, from now on, we assume that $n=1$. In order to get a comparison result, one must impose a control on the size of the jump\$2. This is the reason why (A4) must hold in addition to (A1) and (A2).

Theorem 2 (Yin and Mao (12)) Consider two generators $f_{1}$ and $f_{2}$ verifying (A1), (A2) and (A4). Let $\xi_{\tau}^{1}, \xi_{\tau}^{2} \in L^{2}\left(\Omega, F_{\tau}, P\right)$ be two terminal conditions for BSDEs driven respectively by $f_{1}$ and $f_{2}$. Denote by $\left(Y_{t}^{1}, Z_{t}^{1}, U_{t}^{1}\right)$ and $\left(Y_{t}^{2}, Z_{t}^{2}, U_{t}^{2}\right), t \in[0, \tau]$, the respective solutions of these equations. If $\xi_{\tau}^{1} \leq \xi_{\tau}^{2}$ $P$ - a.s. and $f_{1}\left(t, Y_{t}^{1}, Z_{t}^{1}, U_{t}^{1}\right) \leq f_{2}\left(t, Y_{t}^{1}, Z_{t}^{1}, U_{t}^{1}\right) P-$ a.s., then almost surely $Y_{t}^{1} \leq Y_{t}^{2}$, for all $t \in[0, \tau]$.

Before stating the main theorem, we also need the following lemma. It is a strict comparison theorem for BSDEs with jumps and with the same terminal condition.

Lemma 3 Let the generators $f_{1}$ and $f_{2}$ verify (A1), (A2) and (A4), and let the terminal condition $\xi_{\tau} \in L^{2}\left(\Omega, F_{\tau}, P\right)$. Denote by $\left(Y_{t}^{1}, Z_{t}^{1}, U_{t}^{1}\right)$ and $\left(Y_{t}^{2}, Z_{t}^{2}, U_{t}^{2}\right)$, $t \in[0, \tau]$, the respective solutions of the BSDEs with generators $f_{1}$ and $f_{2}$ and terminal conditions $\xi_{\tau}$. If $f_{2}\left(t, Y_{t}^{1}, Z_{t}^{1}, U_{t}^{1}\right)<f_{1}\left(t, Y_{t}^{1}, Z_{t}^{1}, U_{t}^{1}\right) P-a . s$., then almost surely, $Y_{t}^{2}<Y_{t}^{1}$, for all $t \in[0, \tau)$.

Proof. The proof follows from the same argument as in the proof of the comparison theorem for classical BSDEs of El Karoui et al. (5) (for the jump diffusion case, see the proofs of theorem 3.1 and corollary 3.1 of Yin and Mao (12)).

\section{A converse comparison theorem}

We now introduce the infinite horizon decoupled forward backward stochastic differential equations (FBSDEs, for short) for which we will prove a converse comparison result.

\footnotetext{
${ }^{2}$ See Royer (10) for a counter-example when only Lipschitz continuity is assumed.
} 
For any given $(t, x) \in[0, \infty) \times \mathbb{R}^{m}$, consider the following FBSDEs

$$
\begin{aligned}
X_{s}^{t, x}= & x+\int_{t}^{s} a\left(r, X_{r}^{t, x}\right) d r+\int_{t}^{s} b\left(r, X_{r}^{t, x}\right) d W_{r} \\
& +\int_{t}^{s} \int_{B} c\left(r, X_{r^{-}}^{t, x}, e\right) \widetilde{\mu}(d r, d e), \\
Y_{s}= & h\left(X_{\infty}^{t, x}\right)+\int_{s}^{\infty} f_{1}\left(r, X_{r}^{t, x}, Y_{r}, Z_{r}, \int_{B} U_{r}(e) \gamma_{r}(e)^{\top} \lambda(d e)\right) d r \\
& -\int_{s}^{\infty} Z_{r} d W_{r}-\int_{s}^{\infty} \int_{B} U_{r}(e) \widetilde{\mu}(d r, d e),
\end{aligned}
$$

where $s \in[t, \infty), \gamma$ is assumed to be deterministic and $\gamma:[0, \infty) \times B \rightarrow \mathbb{R}^{1 \times l}$ is $\mathbf{B}\left(\mathbb{R}^{+}\right) \otimes \mathbf{B}$-measurable and satisfies

$\int_{B}\left|\gamma_{t}(e)\right|^{2} \lambda(d e) \leq u_{2}(t)^{2}$, where $u_{2}(t)$ is as in (3) and $\gamma_{t}^{i}(e), i=1, \ldots, l$, is the $i$ th component of $\gamma_{t}(e)$ satisfying $\gamma_{t}^{i}(e) \geq-1$.

From now on, we assume that $a, b, c, f_{1}$ and $h$ in (4) and (5) are deterministic. We assume that $a:[0, \infty) \times \mathbb{R}^{m} \rightarrow \mathbb{R}^{m}, b:[0, \infty) \times \mathbb{R}^{m} \rightarrow \mathbb{R}^{m \times d}$ and $c:$ $[0, \infty) \times \mathbb{R}^{m} \times B \rightarrow \mathbb{R}^{m \times l}$ are continuous and such that they guarantee the existence and uniqueness of a strong solution to (4). We also assume that $X_{\infty}^{t, x}$ exists and that $E\left[\left|X_{\infty}^{t, x}\right|^{2}\right]<\infty$.

Regarding (5), we will suppose that the real valued functions $h: \mathbb{R}^{m} \rightarrow$ $\mathbb{R}$ and $f_{1}:[0, \infty) \times \mathbb{R}^{m} \times \mathbb{R} \times \mathbb{R}^{1 \times d} \times \mathbb{R} \rightarrow \mathbb{R}$ are $\mathbf{B}\left(\mathbb{R}^{m}\right)$-measurable and $\mathbf{B}\left(\mathbb{R}^{+}\right) \otimes \mathbf{B}\left(\mathbb{R}^{m}\right) \otimes \mathbf{B}(\mathbb{R}) \otimes \mathbf{B}\left(\mathbb{R}^{1 \times d}\right) \otimes \mathbf{B}(\mathbb{R})$-measurable, respectively, and that they are Lipschitz continuous with respect to $X$, with Lipschitzian function satisfying (3). Moreover, we assume that for every $X \in \mathbb{R}^{m}, f(t, X, Y, Z, U)=$ $f_{1}\left(t, X, Y, Z, \int_{B} U(e) \gamma_{t}(e)^{\top} \lambda(d e)\right)$ satisfies assumptions (A1), (A2), (A3) and (A4), and we assume that there exists a constant $C$ such that for every $X \in$ $\mathbb{R}^{m},|h(X)| \leq C(1+|X|)$, so that $h\left(X_{\infty}^{t, x}\right) \in L^{2}(\Omega, F, P)$ and (5) admits a unique solution. We denote the solution by $\left(Y_{r}^{t, x, 1}, Z_{r}^{t, x, 1}, U_{r}^{t, x, 1}\right)$, where $Y_{r}^{t, x, 1}$ is adapted and $\left(Z_{r}^{t, x, 1}, U_{r}^{t, x, 1}\right)$ are predictable with respect to the filtration $\left(F_{r}^{t}, r \geq\right.$ $t)$, where $F_{r}^{t}=\sigma\left[W_{s}-W_{t}, \mu((t, s], A) ; t \leq s \leq r, A \in \mathbf{B}\right] \vee N$. Then,

$$
\left(u^{1}(t, x), \chi^{1}(t, x), \zeta^{1}(t, x)\right):=\left(Y_{t}^{t, x, 1}, Z_{t}^{t, x, 1}, \int_{B} U_{t}^{t, x, 1}(e) \gamma_{t}(e)^{\top} \lambda(d e)\right)
$$

is deterministic, and from the uniqueness of solution to (5), it is known (see for example Barles et al. (1), p. 69) that for any $s \in[t, \infty)$,

$$
Y_{s}^{t, x, 1}=Y_{s}^{s, X_{s}^{t, x}, 1}=u^{1}\left(s, X_{s}^{t, x}\right) .
$$

Since the same reasoning holds for $\chi$ and $\zeta$, we similarly have

$$
\begin{aligned}
Z_{s}^{t, x, 1} & =\chi^{1}\left(s, X_{s}^{t, x}\right), \\
\int_{B} U_{s}^{t, x, 1}(e) \gamma_{s}(e)^{\top} \lambda(d e) & =\zeta^{1}\left(s, X_{s-}^{t, x}\right) .
\end{aligned}
$$

We can now state the converse comparison theorem for the infinite horizon decoupled FBSDEs (4)-(5): 
Theorem 4 (Converse comparison theorem) Suppose that $f_{1}$ is continuous and let $a, b, c, f_{1}$ and $h$ satisfy the above assumptions, so that there exists a unique solution to (4) and (5). Denote by $Y_{s}^{t, x, 1}\left(\xi_{v}\right)$ the first component of the solution of (5) with generator $f_{1}$ and terminal condition $\xi_{v}$ at time $v$, where $v$ is a stopping time and $t \leq s<v$. Assume further that $Y_{t}^{t, x, 1}\left(h\left(X_{\infty}^{t, x}\right)\right)=u^{1}(t, x) \in$ $C^{1,2}\left([0, \infty) \times \mathbb{R}^{m}\right)$. If, for any given $(t, x) \in[0, \infty) \times \mathbb{R}^{m}, \exists \delta>0$ such that

$$
Y_{t}^{t, x, 1}\left(u^{1}\left(v, X_{v}^{t, x}\right)\right) \leq Y_{t}^{t, x, 2}\left(u^{1}\left(v, X_{v}^{t, x}\right)\right),
$$

for every stopping time $v$ such that $t \leq v \leq t+\delta$, and where $Y_{t}^{t, x, 2}\left(u^{1}\left(v, X_{v}^{t, x}\right)\right)$ is the first component of the time $t$ solution of a FBSDE with driver $f_{2}$ satisfying the same assumptions as $f_{1}$ and terminal condition $u^{1}\left(v, X_{v}^{t, x}\right)$ at time $v$, then

$$
f_{1}\left(t, x, u^{1}(t, x), \chi^{1}(t, x), \zeta^{1}(t, x)\right) \leq f_{2}\left(t, x, u^{1}(t, x), \chi^{1}(t, x), \zeta^{1}(t, x)\right) .
$$

Proof. By contradiction, suppose that

$$
f_{1}\left(t, x, u^{1}(t, x), \chi^{1}(t, x), \zeta^{1}(t, x)\right)>f_{2}\left(t, x, u^{1}(t, x), \chi^{1}(t, x), \zeta^{1}(t, x)\right) .
$$

By assumption, $f, a, b$ and $c$ are continuous, and $u^{1}$ is assumed to be continuous and smooth, so it follows from the Feynman-Kac formula (see also theorem 3.4 in Barles et al. (1)) that $\chi^{1}$ and $\zeta^{1}$ are continuous functions, since $\chi^{1}(t, x)=$ $u_{x}^{1}(t, x)^{\top} b(t, x)$ and $\zeta^{1}(t, x)=\int_{B}\left(u^{1}(t, x+c(t, x, e))-u^{1}(t, x)\right) \gamma_{t}(e)^{\top} \lambda(d e)$ (the letter $x$ as lower indice indicates differentiation, as usual). As a consequence, there exists $0<\eta<\infty$ such that for any $(s, k) \in[t, \infty) \times \mathbb{R}^{m}$ satisfying $t \leq s \leq t+\eta,|x-k| \leq \eta$,

$$
f_{1}\left(s, k, u^{1}(s, k), \chi^{1}(s, k), \zeta^{1}(s, k)\right)>f_{2}\left(s, k, u^{1}(s, k), \chi^{1}(s, k), \zeta^{1}(s, k)\right) .
$$

Define now the stopping time

$$
\tau:=\inf \left\{s>t:\left|X_{s}^{t, x}-x\right|>\eta\right\} \wedge(t+\eta) \wedge(t+\delta),
$$

and let

$$
\left(\bar{Y}_{s}^{1}, \bar{Z}_{s}^{1}, \bar{U}_{s}^{1}\right):=\left(Y_{s}^{t, x, 1}, Z_{s}^{t, x, 1}, U_{s}^{t, x, 1}\right), t \leq s \leq \tau .
$$

Then, $\left(\bar{Y}_{s}^{1}, \bar{Z}_{s}^{1}, \bar{U}_{s}^{1}\right)$ is solution of the following FBSDE:

$$
\begin{aligned}
\bar{Y}_{s}^{1}= & u^{1}\left(\tau, X_{\tau}^{t, x}\right)+\int_{s}^{\tau} f_{1}\left(r, X_{r}^{t, x}, \bar{Y}_{r}^{1}, \bar{Z}_{r}^{1}, \int_{B} \bar{U}_{r}^{1}(e) \gamma_{r}(e)^{\top} \lambda(d e)\right) d r \\
& -\int_{s}^{\tau} \bar{Z}_{r}^{1} d W_{r}-\int_{s}^{\tau} \int_{B} \bar{U}_{r}^{1}(e) \widetilde{\mu}(d r, d e) .
\end{aligned}
$$

Now, consider the FBSDE

$$
\begin{aligned}
\bar{Y}_{s}^{2}= & u^{1}\left(\tau, X_{\tau}^{t, x}\right)+\int_{s}^{\tau} f_{2}\left(r, X_{r}^{t, x}, \bar{Y}_{r}^{2}, \bar{Z}_{r}^{2}, \int_{B} \bar{U}_{r}^{2}(e) \gamma_{r}(e)^{\top} \lambda(d e)\right) d r(12), \tau \\
& -\int_{s}^{\tau} \bar{Z}_{r}^{2} d W_{r}-\int_{s}^{\tau} \int_{B} \bar{U}_{r}^{2}(e) \widetilde{\mu}(d r, d e)
\end{aligned}
$$


where

$$
\left(\bar{Y}_{s}^{2}, \bar{Z}_{s}^{2}, \bar{U}_{s}^{2}\right):=\left(\bar{Y}_{s}^{t, x, 2}, \bar{Z}_{s}^{t, x, 2}, \bar{U}_{s}^{t, x, 2}\right), t \leq s \leq \tau .
$$

$u^{1}\left(\tau, X_{\tau}^{t, x}\right)$ is the first component of the time $\tau$ solution of a BSDE with coefficient $f_{1}$ and terminal condition $h\left(X_{\infty}^{t, x}\right)$; therefore, $u^{1}\left(\tau, X_{\tau}^{t, x}\right)$ is square integrable, that is, $E\left[u^{1}\left(\tau, X_{\tau}^{t, x}\right)^{2}\right]<\infty$. By assumption on $f_{2}$, it follows that there exists a unique solution to (12).

Taking (6), (7) and (8) into account, together with (11), we can apply the comparison theorem of lemma 3 and get

$$
\bar{Y}_{t}^{1}\left(u^{1}\left(\tau, X_{\tau}^{t, x}\right)\right)>\bar{Y}_{t}^{2}\left(u^{1}\left(\tau, X_{\tau}^{t, x}\right)\right) .
$$

which, by uniqueness, yields that

$$
Y_{t}^{t, x, 1}\left(u^{1}\left(\tau, X_{\tau}^{t, x}\right)\right)>Y_{t}^{t, x, 2}\left(u^{1}\left(\tau, X_{\tau}^{t, x}\right)\right),
$$

which contradicts (9). Therefore, we must have that

$$
f_{1}\left(t, x, u^{1}(t, x), \chi^{1}(t, x), \zeta^{1}(t, x)\right) \leq f_{2}\left(t, x, u^{1}(t, x), \chi^{1}(t, x), \zeta^{1}(t, x)\right) .
$$

\section{References}

[1] Barles, G., Buckdhan, R. and E. Pardoux, 1997. Backward stochastic differential equations and integral-partial differential equations. Stochastics and Stochastics Reports 60, 57-83.

[2] Briand, P., Coquet, F., Hu, Y., Mémin, J. and S. Peng, 2000. A converse comparison theorem for BSDEs and related properties of $g$-expectation. Electronic Communications in Probability 5, 101-117.

[3] Chen, Z., 1998. A property of backward stochastic differential equations. Comptes Rendus de l'Académie des Sciences de Paris, Série I 326, 483-488.

[4] Coquet, F., Hu, Y., Mémin, J. and S. Peng, 2001. A general converse comparison theorem for backward stochastic differential equations. Comptes Rendus de l'Académie des Sciences de Paris, Série I 333, 577-581.

[5] El Karoui, N., Peng, S. and M. C. Quenez, 1997. Backward stochastic differential equations in finance. Mathematical Finance 7, 1-71.

[6] Jiang, L., 2004. Some results on the uniqueness of generators of backward stochastic differential equations. Comptes Rendus de l'Académie des Sciences de Paris, Série I 338, 575-580.

[7] Jiang, L., 2005. Converse comparison theorems for backward stochastic differential equations. Statistics and Probabilty Letters 71, 173-183.

[8] Pardoux, E., Peng, S., 1990. Adapted solution of a backward stochastic differential equation. Systems and Control Letters 14, 55-61. 
[9] Peng, S., 1992. Stochastic Hamilton-Jacobi-Bellman equations. SIAM Journal on Control and Optimization 30, 284-304.

[10] Royer, M., 2006. Backward stochastic differential equations with jumps and related non-linear expectations. Stochastic Processes and their Applications 116, 1358-1376.

[11] Tang, S., Li, X., 1994. Necessary conditions for optimal control of stochastic systems with random jumps. SIAM Journal on Control and Optimization $32,1447-1475$.

[12] Yin, J. and X. Mao, 2008. The adapted solution and comparison theorem for backward stochastic differential equations with Poisson jumps and applications. Journal of Mathematical Analysis and Applications 346, $345-358$.

[13] Yin, J. and R. Situ, 2003. On solutions of forward-backward stochastic differential equation with Poisson jumps. Stochastic Analysis and Applications 23, 1419-1448. 